\title{
Integration by Parts in Differential Summation Form
}

\author{
Hezekiah Seun Adewinbi ${ }^{1, *}$ and Faith Olawoyin ${ }^{2}$ \\ ${ }^{1}$ Department of Mathematics, University of Ibadan, Ibadan, Nigeria \\ e-mail: ayedunseun@gmail.com \\ ${ }^{2}$ Department of Elect/Elect Engineering, University of Ibadan, Ibadan, Nigeria \\ e-mail: olawoyinfaith33@gmail.com \\ *Corresponding author
}

\begin{abstract}
In this paper, we establish general differential summation formulas for integration by parts (IBP), more importantly a powerful tool that promotes exploration and creativity.
\end{abstract}

\section{Introduction}

Definition 1.1. A differential calculus is a branch of mathematics concerned with the determination, properties and application of derivatives and differentials.

Definition 1.2. An integral calculus or antiderivative or primitive assigns numbers to functions in a way that can be described as displacement, area, volume and other concepts that arise by combining infinitesimal data. Integration is one of the two main operations of calculus, with its inverse, differentiation. Integration without limits is called indefinite integrals, while integration with limit is called definite integrals. It is the fundamental theorem of calculus that connects differentiation with the definite integrals.

Definition 1.3 (Fundamental Theorem of Calculus 1). If $f$ is a continuous real-valued function defined on a closed interval $[a, b]$, then, once an antiderivative $F$ of $f$ is known, Received: July 21, 2019; Accepted: August 26, 2019

2010 Mathematics Subject Classification: 00A05.

Keywords and phrases: differential calculus, integral calculus, summation formula, mathematical induction.

Copyright (C) 2019 Hezekiah Seun Adewinbi and Faith Olawoyin. This is an open access article distributed under the Creative Commons Attribution License, which permits unrestricted use, distribution, and reproduction in any medium, provided the original work is properly cited. 
the definite integral of $f$ over that interval is given by

$$
\int_{a}^{b} f(x) d x=\left.F(x)\right|_{a} ^{b}=F(b)-F(a) .
$$

By product rule, if $f(x)$ and $g(x)$ are differentiable functions, then

$$
\frac{d}{d x}(f(x) g(x))=f(x) g^{\prime}(x)+f^{\prime}(x) g(x) .
$$

Integrating both sides, we have

$$
\begin{aligned}
& \int f(x) g^{\prime}(x) d x+\int f^{\prime}(x) g(x) d x=f(x) g(x), \\
& \int f(x) g^{\prime}(x) d x=f(x) g(x)-\int f^{\prime}(x) g(x) d x .
\end{aligned}
$$

Let $u=f(x)$ and $v=g(x)$. Then we obtain the familiar integration by parts formula

$$
\int u d v=u v-\int v d u
$$

\section{Literature Review}

Integral calculus has been the most difficult aspect of mathematics for Secondary School students and preliminary Higher Institution students, most especially integration by parts. Students often find it very difficult in the choice of $u$ and $d v$. Horowitz [3] gives the technique called tabular integration by parts, the method was used to solve some difficult integration by parts, not only that, the method was used to proof some mathematical formulas such as Laplace Transforms Formula, Taylor's Formula, and Residue Theorem for Meromorphic Functions Formula. Knill [4] used the integration by parts formula and tabular integration by parts to solve integration by parts problems, the two results shows that tabular integration by parts is so powerful, not time consuming and reliable. Murty [1] illustrated the procedure of integration by parts with five examples of the type

$$
\int x^{2} \sin x d x, \int e^{x} \sin x d x, \int x^{2} \ln x d x, \int \sin ^{2} x d x, \int \sin 3 x \cos 5 x d x .
$$

The method of tabular integration by parts was used to solve the problems. 
The general formula for integral by parts of the form $\int e^{a x} \cos b x d x$ and $\int e^{a x} \sin b x d x$ is given by

$$
\begin{aligned}
& I_{n}=\int e^{a x} \cos b x d x=\frac{e^{a x}}{a^{2}+b^{2}}(b \sin b x+a \cos b x)+c, \\
& I_{n}=\int e^{a x} \sin b x d x=\frac{e^{a x}}{a^{2}+b^{2}}(a \sin b x-b \cos b x)+c .
\end{aligned}
$$

Equations (2) and (3) can be applied directly to solve problems like

$$
\int e^{2 x} \sin 3 x d x=\frac{e^{2 x}}{13}(2 \sin 3 x-3 \cos 3 x)+c .
$$

Find the Fourier cosine transform of $f(x)=e^{-2 x}+4 e^{-3 x}$

$$
\begin{aligned}
F(s) & =\sqrt{\frac{2}{\pi}} \int_{0}^{\infty} f(x) \cos s x d x \\
& =\sqrt{\frac{2}{\pi}} \int_{0}^{\infty}\left(e^{-2 x}+4 e^{-3 x}\right) \cos s x d x \\
& =\sqrt{\frac{2}{\pi}} \int_{0}^{\infty} e^{-2 x} \cos s x d x+\sqrt{\frac{2}{\pi}} \int_{0}^{\infty} 4 e^{-3 x} \cos s x d x \\
& =2 \sqrt{\frac{2}{\pi}}\left[\frac{1}{s^{2}+4}+\frac{6}{s^{2}+9}\right] .
\end{aligned}
$$

This paper gives some formulas to evaluate integration by parts without thinking about the choose of $u$ and $d v$. Students with no background in integration can also make use of the formulas in solving their integration by parts problems.

\section{Main Results}

Our main results were proved by the principle of mathematical induction.

Theorem 3.1. If $n \in \mathbb{N}$ and $a \neq 0$, then

$$
\int x^{n} \sin a x d x=\sum_{r=0}^{n}{ }^{n} P_{r} x^{n-r} \frac{1}{a^{2 r+1}} \frac{d^{r}}{d x^{r}}(-\cos a x) .
$$


Proof. Let equation (4) be $P(n)$. Consider $P(1)$, we obtain

$$
\begin{aligned}
\int x \sin a x d x & =\sum_{r=0}^{1}{ }^{1} P_{r} x^{1-r} \frac{1}{a^{2 r+1}} \frac{d^{r}}{d x^{r}}(-\cos a x) \\
& =\frac{1}{a^{2}}(-a x \cos a x+\sin a x)+c .
\end{aligned}
$$

Hence, $P(1)$ is true. Assuming, $P(k)$ is true for some $k \in \mathbb{N}$,

$$
\int x^{k} \sin a x d x=\sum_{r=0}^{k}{ }^{k} P_{r} x^{k-r} \frac{1}{a^{2 r+1}} \frac{d^{r}}{d x^{r}}(-\cos a x) .
$$

Consider $P(k+1)$ and apply equation (5), we have the following

$$
\begin{aligned}
\int x^{k+1} \sin a x= & u v-\int v d u \\
& u=x^{k+1}, d u=(k+1) x^{k} \text { and } d v=\sin a x, v=-\frac{1}{a} \cos a x \\
= & -\frac{1}{a} x^{k+1} \cos a x+\frac{k+1}{a} \int x^{k} \cos a x d x \\
= & -\frac{1}{a} x^{k+1} \cos a x+\frac{k+1}{a}\left[\frac{1}{a} x^{k} \sin a x-\int \frac{1}{a} k x^{k-1} \sin a x d x\right] \\
= & -\frac{1}{a} x^{k+1} \cos a x+\frac{k+1}{a^{2}} x^{k} \sin a x-\frac{k(k+1)}{a^{2}} \int x^{k-1} \sin a x d x \\
= & -\frac{1}{a} x^{k+1} \cos a x+\frac{k+1}{a^{2}} x^{k} \sin a x \\
& -\frac{k(k+1)}{a^{2}} \sum_{r=0}^{k-1} k-1 P_{r} x^{k-1-r} \frac{1}{a^{2 r+1}} \frac{d^{r}}{d x^{r}}(-\cos a x) \\
= & \sum_{r=0}^{k+1}{ }^{k+1} P_{r} x^{k+1-r} \frac{1}{a^{2 r+1}} \frac{d^{r}}{d x^{r}}(-\cos a x) .
\end{aligned}
$$

Since $P(k+1)$ is true, also true for all values of $n \in \mathbb{N}$. 
Theorem 3.2. If $n \in \mathbb{N}$ and $a \neq 0$, then

$$
\int x^{n} \cos a x d x=\sum_{r=0}^{n}{ }^{n} P_{r} x^{n-r} \frac{1}{a^{2 r+1}} \frac{d^{r}}{d x^{r}}(\sin a x) .
$$

Proof. See Theorem 3.1.

Theorem 3.3. If $n \in \mathbb{N}$ and $a \neq 0$, then

$$
\int x^{n} \cosh a x d x=\sum_{r=0}^{n}{ }^{n} P_{r} x^{n-r} \frac{1}{a^{2 r+1}}(-1)^{r} \frac{d^{r}}{d x^{r}}(\sinh a x) .
$$

Proof. (By induction) Denote equation (7) by $Q(n)$. Consider $Q(1)$, we have

$$
\begin{aligned}
\int x \cosh a x d x & =\sum_{r=0}^{1}{ }^{1} P_{r} x^{1-r} \frac{1}{a^{2 r+1}}(-1)^{r} \frac{d^{r}}{d x^{r}}(\sinh a x) \\
& =\frac{1}{a^{2}}(a x \sinh a x-\cosh a x)+c .
\end{aligned}
$$

Hence, $Q(1)$ is true. Assuming, $Q(k)$ is true for some $k \in \mathbb{N}$, then

$$
\int x^{k} \cosh a x d x=\sum_{r=0}^{k}{ }^{k} P_{r} x^{k-r} \frac{1}{a^{2 r+1}}(-1)^{r} \frac{d^{r}}{d x^{r}}(\sinh a x) .
$$

Consider $Q(k+1)$ using equation (8), we obtain the following

$$
\begin{aligned}
\int x^{k+1} \cosh a x & =u v-\int v d u \\
& u=x^{k+1}, d u=(k+1) x^{k} \text { and } d v=\cosh a x, v=\frac{1}{a} \sinh a x \\
= & \frac{1}{a} x^{k+1} \sinh a x-\frac{k+1}{a} \int x^{k} \sinh a x d x \\
= & \frac{1}{a} x^{k+1} \sinh a x-\frac{k+1}{a}\left[\frac{1}{a} x^{k} \cosh a x-\int \frac{1}{a} k x^{k-1} \cosh a x d x\right] \\
= & \frac{1}{a} x^{k+1} \sinh a x-\frac{k+1}{a^{2}} x^{k} \cosh a x+\frac{k(k+1)}{a^{2}} \int x^{k-1} \cosh a x d x
\end{aligned}
$$




$$
\begin{aligned}
= & \frac{1}{a} x^{k+1} \sinh a x-\frac{k+1}{a^{2}} x^{k} \cosh a x \\
& +\frac{k(k+1)}{a^{2}} \sum_{r=0}^{k-1}{ }^{k-1} P_{r} x^{k-1-r} \frac{1}{a^{2 r+1}}(-1)^{r} \frac{d^{r}}{d x^{r}}(\sinh a x) \\
= & \sum_{r=0}^{k+1}{ }^{k+1} P_{r} x^{k+1-r} \frac{1}{a^{2 r+1}}(-1)^{r} \frac{d^{r}}{d x^{r}}(\sinh a x) .
\end{aligned}
$$

Since $Q(k+1)$ is true, also true for all values of $n \in \mathbb{N}$.

Theorem 3.4. If $n \in \mathbb{N}$ and $a \neq 0$, then

$$
\int x^{n} \sinh a x d x=\sum_{r=0}^{n}{ }^{n} P_{r} x^{n-r} \frac{1}{a^{2 r+1}}(-1)^{r} \frac{d^{r}}{d x^{r}}(\cosh a x) .
$$

Proof. See Theorem 3.3.

Theorem 3.5. If $n \in \mathbb{N}$ and $a \neq 0$, then

$$
\int x^{n} e^{a x} d x=e^{a x} \sum_{r=0}^{n}(-1)^{r} \frac{1}{a^{r+1}} \frac{d^{r}}{d x^{r}}\left(x^{n}\right) .
$$

Proof. Denote equation (10) by $C(n)$. Consider $C(1)$, we obtain

$$
\int x e^{a x} d x=e^{a x} \sum_{r=0}^{1}(-1)^{r} \frac{1}{a^{r+1}} \frac{d^{r}}{d x^{r}}(x)=\frac{1}{a^{2}}(a x-1)+c .
$$

Hence, $C(1)$ is true. Suppose, $C(k)$ is true for some $k \in \mathbb{N}$, then

$$
\int x^{k} e^{a x} d x=e^{a x} \sum_{r=0}^{k}(-1)^{r} \frac{1}{a^{r+1}} \frac{d^{r}}{d x^{r}}\left(x^{k}\right) .
$$

Consider $C(k+1)$ using equation (11), we obtain

$$
\int x^{k+1} e^{a x}=u v-\int v d u
$$




$$
\begin{aligned}
& u=x^{k+1}, d u=(k+1) x^{k} \text { and } d v=e^{a x}, v=\frac{1}{a} e^{a x} \\
= & \frac{1}{a} x^{k+1} e^{a x}-\frac{(k+1)}{a} \int e^{a x} x^{k} d x \\
= & \frac{1}{a} x^{k+1} e^{a x}-\frac{(k+1)}{a} e^{a x} \sum_{r=0}^{k}(-1)^{r} \frac{1}{a^{r+1}} \frac{d^{r}}{d x^{r}}\left(x^{k}\right) \\
= & e^{a x}\left[\frac{1}{a} x^{k+1}-\frac{(k+1)}{a} \sum_{r=0}^{k}(-1)^{r} \frac{1}{a^{r+1}} \frac{d^{r}}{d x^{r}}\left(x^{k}\right)\right] \\
= & e^{a x} \sum_{r=0}^{k+1}(-1)^{r} \frac{1}{a^{r+1}} \frac{d^{r}}{d x^{r}}\left(x^{k+1}\right) .
\end{aligned}
$$

Hence, $C(k+1)$, also true for all values of $n \in \mathbb{N}$.

Theorem 3.6. If $n \in \mathbb{N}$, then

$$
\int x^{n} \ln x d x=\frac{x^{n+1}}{(n+1)^{2}}[(n+1) \ln x-1] .
$$

Proof. Let equation (12) be $D(n)$. Consider $D(1)$, we have

$$
\int x \ln x d x=\frac{x^{2}}{4}(2 \ln x-1)+c .
$$

Hence, $D(1)$ is true. Suppose that $D(k)$ is true for some $k \in \mathbb{N}$,

$$
\int x^{k} \ln x=\frac{x^{k+1}}{(n+1)^{2}}[(n+1) \ln x-1] .
$$

Consider $D(k+1)$ using equation (13), we obtain the following

$$
\begin{aligned}
\int x^{k+1} \ln x d x= & u v-\int v d u \\
& u=\ln x, v=\frac{1}{x} \text { and } d v=x^{k+1}, v=\frac{1}{k+2} x^{k+2} d x
\end{aligned}
$$




$$
\begin{aligned}
& =\frac{1}{k+2} x^{k+2} \ln x-\frac{1}{k+2} \int x^{k+1} d x \\
& =\frac{1}{k+2} x^{k+2} \ln x-\frac{1}{(k+2)^{2}} x^{k+2} \\
& =\frac{1}{(k+2)^{2}} x^{k+2}[(k+2) \ln x-1] .
\end{aligned}
$$

Since $D(k+1)$ is true, also true for all values of $n \in \mathbb{N}$.

\section{Applications}

Example 4.1. Determine the total charge entering a terminal between $t=1 \mathrm{~s}$ and $t=2 s$ if the current passing the terminal is $i=\left(3 t^{2}-t\right) e^{t}$ Ampere.

Solution. The total charge entering terminal is given by

$$
\begin{aligned}
Q & =\int_{t_{0}}^{t_{1}} i(t) d t \\
& =\int_{1}^{2}\left(3 t^{2}-t\right) e^{t} d t \\
& =\int_{1}^{2} 3 t^{2} e^{t} d t-\int_{1}^{2} t e^{t} d t .
\end{aligned}
$$

By Theorem 3.5, we obtain

$$
\begin{aligned}
Q & =3\left(t^{2}-2 t+2\right) e^{t}-\left.(t-1) e^{t}\right|_{1} ^{2} \\
& =5 e^{2}-3 e \\
& =28.7904350092761 \text { Couloumbs. }
\end{aligned}
$$

Example 4.2. Evaluate $\int x^{2} \sin 3 x d x$.

Solution. Instead of using equation (1), we can apply Theorem 3.1 directly by taking $n=2$ as follows: 


$$
\begin{aligned}
\int x^{n} \sin a x d x & =\sum_{r=0}^{n}{ }^{n} P_{r} x^{n-r} \frac{1}{a^{2 r+1}} \frac{d^{r}}{d x^{r}}(-\cos a x) \\
\int x^{2} \sin 3 x d x & =\sum_{r=0}^{2}{ }^{2} P_{r} x^{2-r} \frac{1}{a^{2 r+1}} \frac{d^{r}}{d x^{r}}(-\cos 3 x) \\
& =\frac{1}{3} x^{2}(-\cos 3 x)+2 x \frac{1}{3^{3}} \frac{d}{d x}(-\cos 3 x)+2 \frac{1}{3^{5}} \frac{d^{2}}{d x^{2}}(-\cos 3 x) \\
& =-\frac{1}{3} x^{2} \cos 3 x+\frac{2}{9} x \sin 3 x+\frac{2}{27} \cos 3 x+c .
\end{aligned}
$$

You can solve the above problem using equation (1) and compare the results.

Example 4.3. Find the energy delivered to an element at $t=3 \mathrm{~s}$ if the current entering its positive terminal is $i=5 \cos 60 \pi t$ Ampere and the voltage across its terminals is $3 t^{3}$.

Solution. Using energy formula and Theorem 3.2, we obtain

$$
\begin{aligned}
E & =\int_{t_{0}}^{t_{1}} I V d t \\
& =\int_{0}^{3} 15 t^{3} \cos (60 \pi t) d t \\
& =\left.\frac{\left(1800 \pi^{2} t^{2}-1\right) \cos (60 \pi t)+60\left(600 \pi^{3} t^{3}-\pi t\right) \sin (60 \pi t)}{144000 \pi^{4}}\right|_{0} ^{3} \\
& =0.0113986 \text { Joules. }
\end{aligned}
$$

Example 4.4. Integrate $\int x^{2} \cos 3 x d x$.

Solution. Theorem 3.2 can be applied directly by taking $n=2$ and $a=3$, we obtain

$$
\int x^{n} \cos a x d x=\sum_{r=0}^{n}{ }^{n} P_{r} x^{n-r} \frac{1}{a^{2 r+1}} \frac{d^{r}}{d x^{r}}(\sin a x)
$$




$$
\begin{aligned}
\int x^{2} \cos 3 x d x & =\sum_{r=0}^{2}{ }^{2} P_{r} x^{2-r} \frac{1}{a^{2 r+1}} \frac{d^{r}}{d x^{r}}(\sin 3 x) \\
& =\frac{1}{3} x^{2} \sin 3 x+2 x \frac{1}{3^{3}} \frac{d}{d x}(\sin 3 x)+2 \frac{1}{3^{5}} \frac{d^{2}}{d x^{2}}(\sin 3 x) \\
& =x^{2} \frac{1}{3}(\sin 3 x)+2 x \frac{1}{3^{3}}(3 \cos 3 x)+2 \frac{1}{3^{5}}(-9 \sin 3 x) \\
& =\frac{1}{3} x^{2} \sin 3 x+\frac{2}{9} x \cos 3 x-\frac{2}{27} \sin 3 x+c .
\end{aligned}
$$

Example 4.5. Evaluate $\int x^{3} e^{2 x} d x$.

Solution. Using Theorem 3.5 with $n=3$ and $a=3$, we obtain

$$
\begin{aligned}
\int x^{n} e^{a x} d x & =e^{a x} \sum_{r=0}^{n}(-1)^{r} \frac{1}{a^{r+1}} \frac{d^{r}}{d x^{r}}\left(x^{n}\right) \\
\int x^{3} e^{2 x} d x & =e^{2 x} \sum_{r=0}^{3}(-1)^{r} \frac{1}{a^{r+1}} \frac{d^{r}}{d x^{r}}\left(x^{3}\right) \\
& =e^{2 x}\left(\frac{1}{2} x^{3}-\frac{3}{4} x^{2}+\frac{3}{4} x-\frac{3}{8}\right) \\
& =\frac{e^{2 x}}{8}\left(4 x^{3}-6 x^{2}+6 x-3\right)+c .
\end{aligned}
$$

Example 4.6. Integrate $\int_{0}^{1} x^{2}(1-x) \cos m \pi x d x$.

Solution. Let

$$
\begin{aligned}
a_{m} & =\int_{0}^{1} x^{2}(1-x) \cos m \pi x d x \\
& =\int_{0}^{1}\left(x^{2} \cos m \pi x-x^{3} \cos m \pi x\right) d x
\end{aligned}
$$




$$
\begin{aligned}
= & 2\left[\sum_{r=0}^{2}{ }^{2} P_{r} x^{2-r} \frac{1}{(m \pi)^{2 r+1}}(-1)^{r} \frac{d^{r}}{d x^{r}}(\sin m \pi x)\right. \\
& \left.-\sum_{r=0}^{3}{ }^{3} P_{r} x^{3-r} \frac{1}{(m \pi)^{2 r+1}}(-1)^{r} \frac{d^{r}}{d x^{r}}(\sin m \pi x)\right] \\
= & \left.2\left[\frac{x^{2}}{m \pi} \sin m \pi x+\frac{2 x}{(m \pi)^{2}} \cos m \pi x-\frac{2}{(m \pi)^{3}} \sin m \pi x\right]\right|_{0} ^{1} \\
& -\left.2\left[\frac{x^{3}}{m \pi} \sin m \pi x+\frac{3 x^{2}}{(m \pi)^{2}} \cos m \pi x-\frac{6 x}{(m \pi)^{3}} \sin m \pi x-\frac{6}{(m \pi)^{4}} \cos m \pi x\right]\right|_{0} ^{1} \\
= & 2\left(\frac{2(-1)^{m}}{(m \pi)^{2}}\right)-2\left(-\frac{3(-1)^{m}}{(m \pi)^{2}}+\frac{6(-1)^{m}}{(m \pi)^{4}}-\frac{6}{(m \pi)^{4}}\right) \\
= & \frac{2(-1)^{m+1}}{(m \pi)^{2}}+\frac{12}{(m \pi)^{4}}\left((-1)^{m}-1\right) .
\end{aligned}
$$

We can see clearly from the above examples that the method of summation is faster and reliable than using equation (1).

\section{Conclusion}

The research shows the direct application of the differential summation formula in solving integration by parts (IBP). Students can solve problems on integration by parts without any basic knowledge in integration but in differentiation. The formula $P(x=r)={ }^{n} C_{r} p^{r} q^{n-r}$ can be used to evaluate expansion, find coefficient of any powers and even the constant term of an expansion. These theorems can also be used to find the coefficient of any power and the constant term in any integration by parts without necessarily integrate completely.

\section{References}

[1] V. N. Murty, Integration by parts, The Two-Year College Mathematics Journal 11(2) (1980), 90-94. https://doi.org/10.2307/3026660 
[2] K. W. Folley, Integration by parts, The American Mathematical Monthly 54(9) (1947), 542-543. https://doi.org/10.2307/2304674

[3] David Horowitz, Tabular integration by parts, The College Mathematics Journal 21(4) (1990), 307-311. https://doi.org/10.1080/07468342.1990.11973325

[4] Oliver Knill, Introduction to Functions and Calculus, Lecture 29, 2012. 